\title{
Long-term mortality in patients with type 2 diabetes undergoing coronary angiography: the impact of glucose-lowering treatment
}

\author{
N. Saleh • P. Petursson • B. Lagerqvist • H. Skúladóttir • \\ A. Svensson • B. Eliasson • S. Gudbjörnsdottir • \\ K. Eeg-Olofsson • A. Norhammar
}

Received: 25 January 2012 / Accepted: 29 March 2012 / Published online: 8 May 2012

(C) Springer-Verlag 2012

\begin{abstract}
Aims/hypothesis The aim of this study was to analyse whether the increased mortality rates observed in insulintreated patients with type 2 diabetes and coronary artery disease are explained by comorbidities and complications. Methods A retrospective analysis of data from two Swedish registries of type 2 diabetic patients $(n=12,515)$ undergoing coronary angiography between the years 2001 and 2009 was conducted. The association between glucose-lowering treatment and long-term mortality was studied after extensive adjustment for cardiovascular- and diabetes-related confounders. Patients were classified into four groups, according to
\end{abstract}

\footnotetext{
N. Saleh $\cdot$ H. Skúladóttir · A. Norhammar $(\bowtie)$

Cardiology Unit, Department of Medicine,

Karolinska University Hospital,

N3:06, Solna,

17176 Stockholm, Sweden

e-mail: anna.norhammar@karolinska.se

e-mail: anna.norhammar@ki.se

P. Petursson

Department of Cardiology, Sahlgrenska University Hospital,

Gothenburg, Sweden

B. Lagerqvist

Department of Medical Sciences, Uppsala University,

Uppsala, Sweden

\author{
A. Svensson \\ Centre of Registers in Region Västra Götaland, \\ Gothenburg, Sweden \\ B. Eliasson · S. Gudbjörnsdottir · K. Eeg-Olofsson \\ Department of Medicine, Sahlgrenska University Hospital, \\ University of Gothenburg, \\ Gothenburg, Sweden
}

glucose-lowering treatment: diet alone; oral therapy alone; insulin in combination with oral therapy; and insulin alone. Results After a mean follow-up time of 4.14 years, absolute mortality rates for patients treated with diet alone, oral therapy alone, insulin in combination with oral therapy and insulin alone were $19.2 \%, 17.4 \%, 22.9 \%$ and $28.1 \%$, respectively. Compared with diet alone, insulin in combination with oral therapy (HR 1.27; 95\% CI 1.12, 1.43) and insulin alone (HR 1.62; 95\% CI 1.44, 1.83) were associated with higher mortality rates. After adjustment for baseline differences, insulin in combination with oral glucoselowering treatment (HR 1.22; 95\% CI 1.06, 1.40; $p<$ 0.005 ) and treatment with insulin only (HR $1.17 ; 95 \% \mathrm{CI}$ $1.02,1.35 ; p<0.01)$ remained independent predictors for long-term mortality.

Conclusions/interpretation Type 2 diabetes patients treated with insulin and undergoing coronary angiography have a higher long-term mortality risk after adjustment for measured confounders. Further research is needed to evaluate the optimal glucose-lowering treatment for these high-risk patients.

Keywords Coronary angiography · Coronary artery disease $\cdot$ Diabetes $\cdot$ Insulin $\cdot$ Mortality $\cdot$ Treatment
Abbreviations
CABG Coronary artery bypass graft
CAD Coronary artery disease
DIGAMI-2 Diabetes Mellitus Insulin-Glucose Infusion in Acute Myocardial Infarction 2
NDR National Diabetes Register
PCI Percutaneous coronary intervention
SCAAR Swedish Coronary Angiography
Angioplasty Registry 


\section{Introduction}

Diabetes is associated with a high prevalence of coronary artery disease (CAD) and an unfavourable prognosis. Patients with diabetes have at least a doubled risk for acute myocardial infarction compared with those without diabetes, after adjustment for traditional risk factors [1]. Further, diabetes is a strong independent predictor of long-term mortality in patients with established CAD [2], despite the use of modern revascularisation treatments [3, 4].

Considering the detrimental cardiovascular effects of chronic hyperglycaemia, it seems reasonable to assume that strict glucose-lowering therapy is beneficial. Indeed, intensive glycaemic control in patients with newly diagnosed diabetes has been shown to improve cardiovascular outcome and survival [5]. However, randomised controlled trials have not confirmed the beneficial effect of intensive glycaemic control in patients with established diabetes and high cardiovascular risk [6-8]. A meta-analysis of five randomised trials showed that intensive compared with standard glycaemic control modestly reduced coronary events but had no significant effect on mortality [9].

In addition to uncertainty regarding optimal glycaemic control, the preferred glucose-lowering therapy for patients with CAD remains to be clarified. In post-hoc analyses of the Diabetes Mellitus Insulin-Glucose Infusion in Acute Myocardial Infarction 2 (DIGAMI-2) trial, insulin-based treatment in patients suffering myocardial infarction was associated with increased risk of non-fatal myocardial infarction and stroke, whereas treatment with metformin was found to be more beneficial [10]. A recent report from the Swedish Coronary Angiography Angioplasty Registry (SCAAR) showed that long-term mortality after percutaneous coronary intervention (PCI) was highest among those treated with insulin [3]. Since these later data are from epidemiological analyses and not from a randomised trial of insulin, the potentially harmful cardiovascular effect of insulin warrants more thorough investigation.

We hypothesise that insulin treatment is a marker of more advanced diabetes rather than a direct cause of adverse outcome in patients with CAD. In this study we attempt to elucidate this question by merging data from two large national registries and thus incorporating important diabetes-related variables related to increased insulin use and complications.

\section{Methods}

\section{The SCAAR}

The SCAAR (www.ucr.uu.se/scaar/index.htm) collects data on all consecutive patients undergoing coronary angiography and PCI at all 29 centres that perform coronary angiography and PCI in Sweden. Data have been monitored and verified at all hospitals since 2001 by annually comparing 50 entered variables in 20 randomly selected interventions per hospital with the patients' hospital records. The overall correspondence in data during the study period was $95.2 \%$. The registry is sponsored by the Swedish health authorities and is independent of commercial funding.

\section{The National Diabetes Register}

The Swedish National Diabetes Register (NDR) was initiated in 1996 as a tool for local quality assurance in diabetes care at a national level and has previously been described in detail [11]. It is administered from the Centre of Registers in Region Västra Götaland, Gothenburg, Sweden.

Reporting to the register is not mandatory, but all hospital diabetes outpatient clinics and primary healthcare centres are encouraged to do so. More than 260,000 patients were reported to the registry in 2009, which is estimated to be $70 \%$ of all patients with diabetes in Sweden. Annual reporting of 24 variables to the NDR is carried out by trained physicians and nurses via either the Internet or clinical records databases, with information collected during patient visits at hospital outpatient clinics and primary healthcare centres nationwide. All included patients provided informed consent to become registered before inclusion.

Present patient population and merging of data

Data were merged from the SCAAR and NDR for all patients who underwent coronary angiography between 1 January 2001 and 31 December 2009. In the case of multiple registrations data were only collected from the patient's first coronary angiography during the study period. Follow-up data on mortality and any hospitalisations were obtained by merging the data with the Swedish National Population Registry and the Swedish Registry on Hospital Diagnosis up to 31 December 2009. Patients defined as having type 1 diabetes in the NDR were excluded from the present analysis. All variables from the diabetes registry were collected before the angiography event, but only patients with an $\mathrm{HbA}_{1 \mathrm{c}}$ result recorded no longer than 3 years before the angiography were included. Information on amputation and dialysis before the angiography event was obtained from the Swedish Registry on Hospital Diagnosis.

\section{Definitions}

Diabetes The epidemiological definition of type 2 diabetes used in this study was: a patient treated with diet alone or oral glucose-lowering agent alone, or age at onset of diabetes $\geq 40$ years and treatment with insulin either alone or in combination with an oral glucose-lowering agent. The 
epidemiological definition of type 1 diabetes used in this study and thus for exclusion from the present analysis was: a patient treated with insulin and diabetes diagnosed before the age of 30 years. Patients with age at onset of 30-39 years and insulin treatment were excluded from this study as it was difficult to classify them epidemiologically as having type 1 diabetes, latent autoimmune diabetes of adulthood or type 2 diabetes; such patients constituted only a small proportion of all patients with diabetes.

Previous hospitalisation A new variable was created to identify a severely diseased patient who had been hospitalised for any reason at a Swedish hospital during the last 3 years before angiography. This was considered a way to identify patients with diabetes and severe disease, and in probable need of insulin because of this hospitalised illness, who were not covered by other variables in the two registries.

Laboratory analysis and recorded variables All laboratory analyses were carried out at local laboratories. The data for blood pressure, lipids, creatinine, blood glucose, weight and BMI were collected from the NDR visit before the angiography event. If missing from the NDR, data were searched for in the SCAAR. $\mathrm{HbA}_{1 \mathrm{c}}$ results were quality assured nationwide by regular calibration with the HPLC Mono-S method. In this study, all $\mathrm{HbA}_{1 \mathrm{c}}$ values were converted to the DCCT standard values using the following formula: $\mathrm{HbA}_{1 \mathrm{c}}(\mathrm{DCCT})=0.923 \times \mathrm{HbA}_{1 \mathrm{c}}($ Mono-S $)+1.345\left(R^{2}=\right.$ 0.998) [12]. For baseline $\mathrm{HbA}_{1 \mathrm{c}}$ we used the most recent value measured up to 3 years before the coronary angiography. $\mathrm{HbA}_{1 \mathrm{c}}$ values older than 3 years were rare and considered too old and therefore too inaccurate to relate to the coronary findings and outcome. The mean time from $\mathrm{HbA}_{1 \mathrm{c}}$ to PCI performance was 298 days (SD 258 days) and the median time with interquartile range was 221 days (99420 days). Cumulative microalbuminuria was collected from the NDR and defined as urine albumin excretion $>20 \mu \mathrm{g} / \mathrm{min}$ in two out of three consecutive tests.

Angiography findings The extent of coronary artery disease was classified according to the number (one, two or three, or left main) of major coronary arteries with significant luminal stenosis $\geq 50 \%$.

Outcome The primary outcome was long-term mortality, defined as death from any cause during the follow-up period.

\section{Statistical analysis}

Baseline data were recorded on the day of the angiography event or taken from the last previous contact recorded in the NDR. Patients were divided into four groups according to glucose-lowering treatment at baseline: insulin alone; insulin in combination with oral therapy; oral therapy alone; and diet alone. Comparisons between the groups were made for: background characteristics; diabetes; angiography event; treatment at baseline; and outcome. Differences between groups were assessed by means of $\chi^{2}$ test for categorical variables and ANOVA for continuous variables. Long-term mortality was adjusted for differences in background and angiography characteristics in a multivariable proportional Cox regression model (age, angiography indication, sex, hospital, previous myocardial infarction, previous PCI, previous coronary artery bypass graft $[\mathrm{CABG}]$, previous heart failure, previous renal insufficiency and dialysis, known cancer, previous stroke, previous peripheral artery disease and amputation, hypertension, treatment of hyperlipidaemia, smoking, angiography findings, year of SCAAR registration, retinopathy, diabetes duration and $\left.\mathrm{HbA}_{1 \mathrm{c}}\right)$. The term 'previous hospitalisation' was also included in the adjustments. BMI was not included in the main multivariable model because of the large number of missing values, but a separate subgroup analysis of obese and overweight individuals was performed. Patients with missing data for a variable in the NDR were considered to be free from the complication. All known variables with possible clinical importance recorded in the SCAAR and NDR were forced into the statistical model.

Information was available for 10,952 individuals for cumulative microalbuminuria and estimated creatinine clearance (calculated by the Cockcroft-Gault formula: creatinine clearance $[\mathrm{ml} / \mathrm{min}]=[\{140-$ age $\} \times$ weight $\times 1.23 \times$ $\{0.85$ if female $\}] /$ creatinine $[\mu \mathrm{mol} / 1]$ ) and was entered in a final multivariable proportional Cox regression model. All analyses were conducted using the SPSS statistical program (version 19.0; SPSS, Chicago, IL, USA).

\section{Ethical considerations}

All patients were informed of their participation in the SCAAR and the NDR. The merging of the registries was performed by the Centre for Epidemiology at the Swedish National Board of Health and Welfare and was approved by the local ethics committee at Gothenburg University.

\section{Results}

Between 2001 and 2009, 294,951 patients underwent coronary angiography for the evaluation of CAD and were recorded in the SCAAR. Of these patients, $18.5 \%$ had diabetes. In all, data for 14,080 patients with type 2 diabetes and a first recorded angiography were available from both registries. The mean age of the study population was 68.5 (SD 9.3) years and 62\% were male. Complete data on 
Table 1 Baseline characteristics and ongoing treatment in patients with type 2 diabetes with a first recorded angiography in SCAAR, according to diabetes treatment

\begin{tabular}{|c|c|c|c|c|c|}
\hline Characteristic or risk factor & $\begin{array}{l}\text { No treatment } \\
n=2,428\end{array}$ & $\begin{array}{l}\text { Oral treatment } \\
n=5,051\end{array}$ & $\begin{array}{l}\text { Insulin }+ \text { oral } \\
\text { treatment } n=2,803\end{array}$ & $\begin{array}{l}\text { Insulin treatment } \\
n=2,233\end{array}$ & $p$ value \\
\hline \multicolumn{6}{|l|}{ Baseline characteristic } \\
\hline Age (years [mean, SD]) & $69.0(9.8)$ & $68.0(9.6)$ & $68.1(8.3)$ & $69.2(8.8)$ & $<0.0001$ \\
\hline Male $(\%)$ & 65.0 & 68.4 & 64.1 & 65.1 & 0.0001 \\
\hline BMI $\left(\mathrm{kg} / \mathrm{m}^{2}\right)$ & $28.2(5.2)$ & $28.6(4.5)$ & $30.3(4.9)$ & $28.6(7.8)$ & $<0.0001$ \\
\hline Weight (kg) & $82.9(15.5)$ & $84.7(15.6)$ & $90.3(15.6)$ & $83.8(16.1)$ & $<0.0001$ \\
\hline \multicolumn{6}{|l|}{ Previous disease (\%) } \\
\hline Hypertension (treated) & 68.1 & 73.0 & 78.4 & 73.4 & $<0.0001$ \\
\hline Hyperlipidaemia (treated) & 59.2 & 66.1 & 73.7 & 69.1 & $<0.0001$ \\
\hline CABG & 10.7 & 10.5 & 13.7 & 15.3 & $<0.0001$ \\
\hline PCI & 15.1 & 14.6 & 16.0 & 17.8 & 0.017 \\
\hline Myocardial infarction & 30.9 & 30.0 & 35.3 & 40.2 & $<0.0001$ \\
\hline Heart failure (hospitalised) & 12.9 & 11.6 & 16.4 & 23.9 & $<0.0001$ \\
\hline Stroke (hospitalised) & 9.5 & 8.2 & 11.0 & 11.4 & $<0.0001$ \\
\hline Renal insufficiency (hospitalised) & 1.6 & 0.8 & 2.1 & 7.3 & $<0.0001$ \\
\hline Smoker (current) & 11.7 & 12.5 & 10.7 & 9.6 & 0.002 \\
\hline Peripheral artery disease (hospitalised) & 5.4 & 4.3 & 6.7 & 11.1 & $<0.0001$ \\
\hline Dialysis & 0.4 & 0.2 & 0.3 & 2.5 & $<0.0001$ \\
\hline Amputation (hospitalised) & 1.0 & 0.9 & 1.3 & 2.2 & 0.0001 \\
\hline Cancer (hospitalised) & 3.2 & 2.8 & 2.5 & 4.1 & 0.009 \\
\hline \multicolumn{6}{|l|}{ Diabetes-related variables } \\
\hline $\mathrm{HbA}_{1 \mathrm{c}}(\%)$ & $6.5(2.2)$ & $7.1(2.4)$ & $7.8(2.5)$ & $7.7(2.6)$ & $<0.0001$ \\
\hline $\mathrm{HbA}_{1 \mathrm{c}}(\mathrm{mmol} / \mathrm{l})$ & $47.5(0.5)$ & $54.1(2,7)$ & $61.7(3.8)$ & $60.7(4.9)$ & $<0.0001$ \\
\hline Diabetes duration (years) & $5.6(5.8)$ & $8.6(6.1)$ & $13.6(6.6)$ & $15.0(8.3)$ & $<0.0001$ \\
\hline Creatinine $(\mu \mathrm{mol} / \mathrm{l})$ & $87.8(34.6)$ & $84.9(28.1)$ & $88.7(37.1)$ & $107.4(81.9)$ & $<0.0001$ \\
\hline Systolic blood pressure $(\mathrm{mmHg})$ & $142(18)$ & $142(18)$ & $141(18)$ & $142(19)$ & 0.498 \\
\hline Diastolic blood pressure $(\mathrm{mmHg})$ & $77(10)$ & $77(10)$ & $76(10)$ & $75(10)$ & $<0.0001$ \\
\hline Pulse pressure $(\mathrm{mmHg})$ & $64(17)$ & $65(16)$ & $65(16)$ & $67(17)$ & 0.002 \\
\hline HDL-cholesterol (mmol/1) & $1.28(0.40)$ & $1.24(0.36)$ & $1.20(0.37)$ & $1.32(0.46)$ & $<0.0001$ \\
\hline Triacylglycerols $(\mathrm{mmol} / \mathrm{l})$ & $1.78(1.02)$ & $2.02(1.21)$ & $2.13(1.41)$ & $1.88(1.36)$ & $<0.0001$ \\
\hline Total cholesterol (mmol/1) & $4.99(1.03)$ & $4.93(1.04)$ & $4.74(1.02)$ & $4.87(1.07)$ & $<0.0001$ \\
\hline Low physical activity level (\%) & 13.8 & 14.2 & 22.1 & 22.3 & $<0.0001$ \\
\hline High physical activity level (\%) & 35.5 & 27.4 & 22.8 & 28.2 & $<0.0001$ \\
\hline Retinopathy (\%) & 13.0 & 24.3 & 50.3 & 53.9 & $<0.0001$ \\
\hline Only angiography (no PCI) (\%) & 56.7 & 57.1 & 59.0 & 59.2 & 0.142 \\
\hline Angiography findings (\%) & & & & & $<0.0001^{\mathrm{a}}$ \\
\hline Normal & 22.1 & 20.6 & 18.7 & 17.5 & \\
\hline One-vessel disease & 23.6 & 23.8 & 21.8 & 19.6 & \\
\hline Two-vessel disease & 20.9 & 19.8 & 20.0 & 20.8 & \\
\hline Three-vessel disease & 21.7 & 26.2 & 27.8 & 29.9 & \\
\hline Left main stem disease & 11.7 & 9.5 & 11.4 & 12.0 & \\
\hline Angiography decision (\%) & & & & & $<0.001^{\mathrm{a}}$ \\
\hline No coronary intervention & 35.1 & 34.7 & 34.7 & 36.0 & \\
\hline CABG & 17.7 & 19.2 & 19.9 & 19.0 & \\
\hline PCI & 47.2 & 46.1 & 45.4 & 45.0 & \\
\hline Indication (\%) & & & & & $0.475^{\mathrm{a}}$ \\
\hline Stable angina & 28.2 & 31.0 & 31.9 & 26.6 & \\
\hline NSTE-ACS & 44.7 & 43.6 & 43.8 & 46.8 & \\
\hline STEMI & 10.6 & 10.0 & 8.5 & 10.3 & \\
\hline Previous hospitalisation (days) & $6.8(18.3)$ & $5.7(13.0)$ & $7.6(16.1)$ & $11.9(31.3)$ & $<0.0001$ \\
\hline
\end{tabular}

Data are presented as percentages and mean (SD) unless otherwise indicated

${ }^{\text {a }}$ Overall

NSTE-ACS, non-ST-elevation acute coronary syndrome; STEMI, ST-elevation myocardial infarction 
baseline characteristics for the adjusted survival analysis were available for 12,515 of the patients from the original cohort. Baseline characteristics of the patients, presented according to previous glucose-lowering treatment, are summarised in Table 1. Patients receiving insulin (with or without oral treatment) more commonly had established cardiovascular disease, longer diabetes duration, higher prevalence of diabetes complications and worse glucose control. Further, insulin treatment was associated with more advanced CAD, a lower likelihood of completely normal angiography, and a higher likelihood of having disease affecting three vessels. This scenario was even more often present in patients on insulin only, indicating that the patients on insulin only had the highest level of diseased state.

\section{Mortality}

During a mean follow-up period of 4.14 (SD 2.0) years, there were 3,093 (22\%) deaths from any cause. Absolute mortality rates at 6 months after the angiography were $5.0 \%$, $4.6 \%, 7.0 \%$ and $8.4 \%(p<0.0001)$ in patients with diet alone, oral therapy alone, insulin in combination with oral glucose-lowering drugs and insulin only. The corresponding figures after a mean follow-up of 4.14 years were $19.2 \%$, $17.4 \%, 22.9 \%$ and $28.1 \%$, respectively (Fig. 1a). Compared with diet alone, insulin in combination with oral therapy (HR 1.27; 95\% CI 1.12, 1.43) and insulin alone (HR 1.62; $95 \%$ CI $1.44,1.83$ ) were associated with higher long-term mortality. After adjusting for baseline differences, insulin in combination with oral glucose-lowering treatment (HR 1.22; $95 \%$ CI 1.06, 1.40; $p<0.005$ ) and treatment with insulin only (HR 1.17; 95\% CI 1.02, 1.35; $p<0.01$ ) remained independent predictors for long-term mortality (Fig. $1 \mathrm{~b}$ and Table 3). Predictors for mortality are shown in Table 2. Evidence of myocardial damage, as heart failure, and diabetes complications, particularly renal complications, was strongly predictive. Glucose control was a weaker, but still an independent, predictor for mortality.

A separate analysis was performed in the subgroup of patients with data available for estimated creatinine clearance and microalbuminuria $(n=10,952)$, thus even including adjustments for renal complications. Compared with the reference group (diet alone), treatment with insulin in combination with oral therapy (adjusted HR 1.35; 95\% CI 1.15, $1.58 ; p<0.001$ ) and insulin treatment alone (adjusted HR $1.23 ; 95 \%$ CI $1.04,1.46 ; p<0.016)$ were associated with higher long-term mortality. Oral therapy alone did not predict long-term mortality (HR 1.04; 95\% CI 0.91, 1.19; $p=0.578$ ).

Subgroup analyses in patients with known BMI $(n=$ 11,088 ) are shown in Table 3. Unadjusted HRs for mortality were increased in insulin-treated patients in all BMI groups. After adjustments for other comorbidities, the association
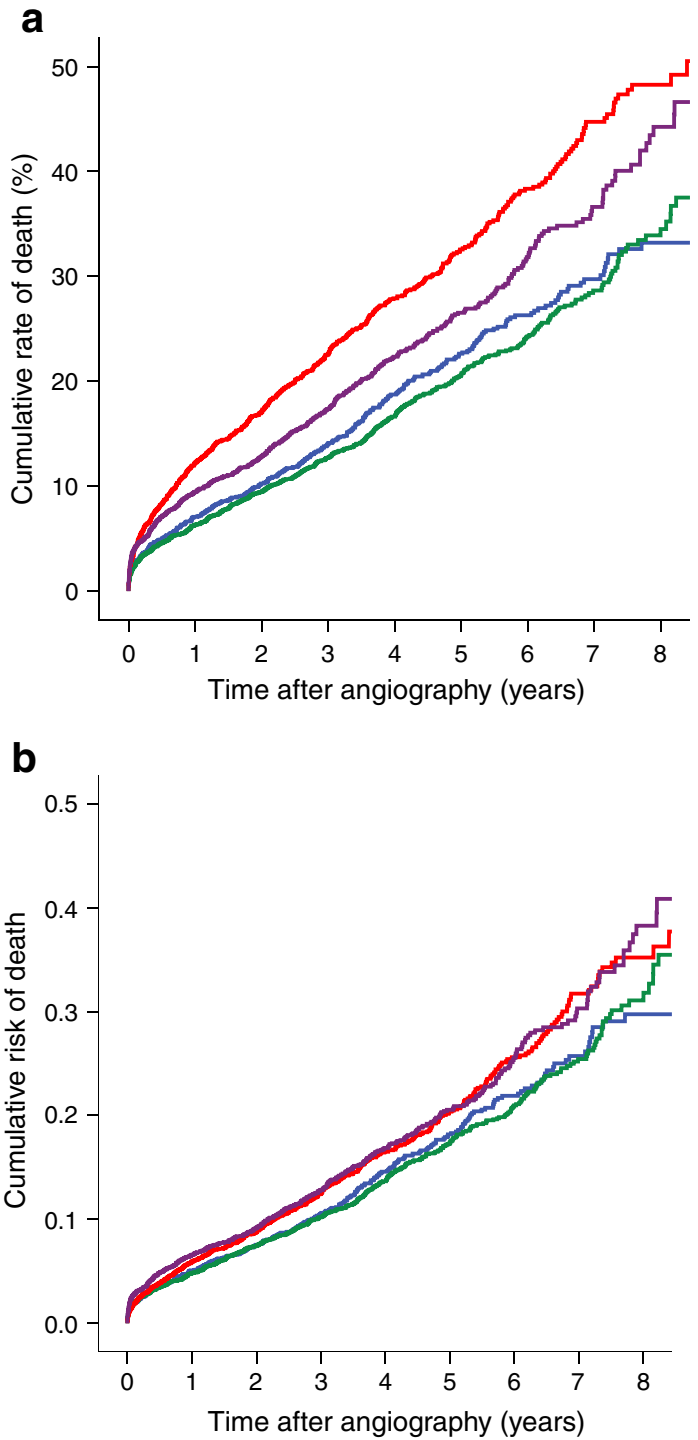

Fig. 1 Long-term mortality after angiography in patients with type 2 diabetes by glucose-lowering treatment: (a) unadjusted and (b) adjusted. Blue, diet $(n=2,428)$; green, oral glucose-lowering drugs $(n=$ $5,051)$; purple, insulin + oral glucose-lowering drugs $(n=2,803)$; and red, insulin treatment only $(n=2,233)$

remained statistically significant in patients with BMI $<30 \mathrm{~kg} / \mathrm{m}^{2}$ but not in the obese patients (BMI $>30 \mathrm{~kg} / \mathrm{m}^{2}$ ). However, when BMI was entered into the multivariable model for those with known BMI, this factor was not found to be an independent predictor of mortality (HR 1.00; $95 \%$ CI $0.99,1.01)$.

\section{Discussion}

In this register-based retrospective cohort study with extensively recorded cardiovascular- and diabetes-related risk variables, insulin treatment with or without oral glucoselowering therapy was associated with higher long-term 
Table 2 Unadjusted and adjusted predictors of mortality
NSTE-ACS, non-ST-elevation acute coronary syndrome; STEMI, ST-elevation myocardial infarction

\begin{tabular}{|c|c|c|c|c|}
\hline \multirow[t]{2}{*}{ Variable } & \multicolumn{2}{|c|}{ Unadjusted } & \multicolumn{2}{|c|}{ Adjusted } \\
\hline & HR & $95 \% \mathrm{CI}$ & HR & $95 \% \mathrm{CI}$ \\
\hline Age (each year) & 1.07 & $1.07,1.08$ & 1.06 & $1.06,1.07$ \\
\hline Sex (\% male) & 0.99 & $0.92,1.08$ & 0.96 & $0.88,1.05$ \\
\hline Hypertension (treated) & 1.09 & $0.96,1.19$ & 0.98 & $0.90,1.08$ \\
\hline Hyperlipidaemia (treated) & 0.86 & $0.79,0.93$ & 0.86 & $0.79,0.94$ \\
\hline $\mathrm{HbA}_{1 \mathrm{c}}($ each \%) & 1.06 & $1.03,1.10$ & 1.04 & $1.01,1.08$ \\
\hline Smoker (current) & 0.98 & $0.84,1.10$ & 1.58 & $1.34,1.86$ \\
\hline \multicolumn{5}{|l|}{ Previous disease } \\
\hline Myocardial infarction & 2.08 & $1.92,2.24$ & 1.33 & $1.21,1.45$ \\
\hline PCI & 1.41 & $1.27,1.56$ & 1.29 & $1.15,1.44$ \\
\hline CABG & 1.80 & $1.63,1.99$ & 1.14 & $1.02,1.27$ \\
\hline Heart failure & 3.12 & $2.88,3.40$ & 2.02 & $1.84,2.22$ \\
\hline Stroke & 1.86 & $1.68,2.08$ & 1.26 & $1.13,1.14$ \\
\hline Renal insufficiency & 3.66 & $3.11,4.32$ & 1.51 & $1.23,1.86$ \\
\hline PAD & 2.47 & $2.20,2.78$ & 1.46 & $1.28,1.65$ \\
\hline Dialysis & 4.72 & $3.60,6.18$ & 1.85 & $1.32,2.60$ \\
\hline Cancer & 1.90 & $1.58,2.28$ & 1.34 & $1.11,1.62$ \\
\hline Diabetes duration (each year) & 1.04 & $1.03,1.04$ & 1.006 & $1.001,1.012$ \\
\hline Retinopathy & 1.53 & $1.35,1.73$ & 1.25 & $1.10,1.43$ \\
\hline Amputation & 2.40 & $1.91,3.03$ & 1.47 & $1.16,1.87$ \\
\hline \multicolumn{5}{|l|}{ Angiography findings } \\
\hline Normal & 1 & & & \\
\hline One vessel & 1.20 & $1.04,1.40$ & 1.11 & $0.95,1.30$ \\
\hline Two vessel & 1.56 & $1.35,1.80$ & 1.27 & $1.09,1.48$ \\
\hline Three vessel & 2.20 & $1.93,2.51$ & 1.60 & $1.38,1.85$ \\
\hline Left main vessel & 2.70 & $2.33,3.14$ & 1.79 & $1.52,2.12$ \\
\hline \multicolumn{5}{|l|}{ Angiography indication } \\
\hline Stable angina & 1 & & & \\
\hline NSTE-ACS & 1.70 & $1.54,1.88$ & 1.39 & $1.25,1.54$ \\
\hline STEMI & 2.94 & $2.57,3.35$ & 2.57 & $2.22,2.97$ \\
\hline Previous hospitalisation (each day) & 1.004 & $1.003,1.004$ & 1.003 & $1.002,1.004$ \\
\hline
\end{tabular}

mortality in patients with diabetes undergoing coronary angiography. Patients treated with insulin alone had the highest mortality rate, with $8.4 \%$ mortality after 6 months and $28.14 \%$ after a mean of 4.14 years. These patients were also shown to have more complications but, after extensive adjustments for potential confounders, treatment with insulin remained an independent predictor for mortality. In patients treated with insulin in combination with oral glucose-lowering drugs, absolute mortality rates were lower and these patients less commonly had complications compared with patients using insulin alone. Despite this, combined treatment was even more strongly related to mortality after adjusting for confounders in comparison with diet or oral glucose-lowering treatment alone.

These findings are in agreement with results from registry studies and subgroup analyses of clinical trials that have raised concerns about the possibly harmful effects of exogenous insulin treatment in patients with CAD [3, 13, 14]. In the Euro Heart Survey on Diabetes and the Heart, insulin treatment was associated with roughly doubled adjusted 1 year mortality risk compared with oral glucose-lowering treatment [13]. An analysis of data from SCAAR showed a higher long-term mortality rate after first PCI in patients with diabetes compared with those without. Moreover, the mortality risk was higher for insulin-treated than for noninsulin-treated diabetes (adjusted RR 2.03, 95\% CI 1.8, 2.28 and $1.45,95 \%$ CI $1.28,1.64$, respectively) [3]. The DIGAMI-2 trial studied different glucose-lowering modalities in patients with diabetes and acute myocardial infarction and showed no differences in mortality or glucose control between the randomised treatment arms [15]. A post hoc analysis of data from the DIGAMI-2 trial revealed that the 
Table 3 Unadjusted and adjusted HRs for mortality by glucose-lowering treatment and in obese and non-obese individuals separately

\begin{tabular}{|c|c|c|c|c|}
\hline \multirow[t]{2}{*}{ Diabetes treatment } & \multicolumn{2}{|c|}{ Unadjusted } & \multicolumn{2}{|c|}{ Adjusted $^{\mathrm{a}}$} \\
\hline & HR & $95 \% \mathrm{CI}$ & HR & $95 \% \mathrm{CI}$ \\
\hline \multicolumn{5}{|c|}{ All patients $(n=12,515)$} \\
\hline Diet & 1 & & & \\
\hline Oral & 0.92 & $0.82,1.04$ & 0.97 & $0.86,1.10$ \\
\hline Oral + insulin & 1.27 & $1.12,1.43$ & 1.22 & $1.06,1.40$ \\
\hline Insulin & 1.62 & $1.44,1.83$ & 1.17 & $1.02,1.35$ \\
\hline \multicolumn{5}{|c|}{ Patients with known BMI $(n=11,088)$} \\
\hline Diet & 1 & & & \\
\hline Oral & 0.80 & $0.87,1.12$ & 1.01 & $0.89,1.15$ \\
\hline Oral + insulin & 1.34 & $1.17,1.53$ & 1.23 & $1.05,1.43$ \\
\hline Insulin & 1.74 & $1.52,1.98$ & 1.22 & $1.04,1.43$ \\
\hline \multicolumn{5}{|l|}{$\mathrm{BMI}<30 \mathrm{~kg} / \mathrm{m}^{2}$} \\
\hline Diet & 1 & & & \\
\hline Oral & 0.99 & $0.85,1.15$ & 1.04 & $0.89,1.22$ \\
\hline Oral + insulin & 1.27 & $1.07,1.50$ & 1.28 & $1.05,1.55$ \\
\hline Insulin & 1.46 & $1.25,1.71$ & 1.22 & $1.004,1.48$ \\
\hline \multicolumn{5}{|l|}{$\mathrm{BMI}>30 \mathrm{~kg} / \mathrm{m}^{2}$} \\
\hline Diet & 1 & & & \\
\hline Oral & 1.02 & $0.80,1.30$ & 1.00 & $0.78,1.29$ \\
\hline Oral + insulin & 1.66 & $1.31,2.10$ & 1.20 & $0.91,1.57$ \\
\hline Insulin & 2.52 & $1.97,3.21$ & 1.29 & $0.96,1.72$ \\
\hline
\end{tabular}

risk of a non-fatal cardiovascular event was higher in patients treated with insulin (adjusted HR 1.71; 95\% CI $1.25,2.35 ; p=0.0008)$. It is noteworthy that this relationship between insulin and cardiovascular events tended to be strongest among those patients who, according to the study protocol, were randomised to insulin-based treatment (HR 2.07; 95\% CI 1.37, 3.14; $p=0.0006$ ) [10].

There are several possible explanations for the present finding. Whether the insulin directly contributes to the worse prognosis, or whether treatment is just a marker of more advanced diabetes, has been debated. Several potentially harmful effects of insulin have been identified. In a number of studies hypoglycaemia has been associated with worse outcome in patients with myocardial infarction [16-19]. However, hypoglycaemia in patients given insulin has not been shown to predict poor prognosis in this patient population [20, 21]. Further, insulin has been shown to be associated with endothelial dysfunction [22] and it is known that patients treated with insulin often have reduced responsiveness to antiplatelet treatment [23]. Whether platelet dysfunction in these patients is a result of insulin treatment, insulin resistance or both, is not clear. Weight gain is another problem related to insulin treatment. However, we did not find an increased mortality risk in obese patients treated with insulin after adjusting for other risk factors, whereas the risk increase remained in the insulin-treated non-obese patients, even after adjustment for other risk factors. Nor was BMI an independent predictor for mortality in the adjusted model that included patients with recorded BMI.

It is important to note that the present study is based on an analysis of observational data and therefore we cannot rule out residual confounding - that differences between groups other than those recorded at baseline may have influenced the results. Extensive adjustments for measured confounders were possible by merging the two registries, where one focused on diabetes variables and the other on cardiovascular variables, thus, to a large extent, limiting the confounding effects of diabetes severity and cardiovascular disease. By excluding patients with type 1 diabetes, the cohort receiving insulin treatment were even less confounded than previously reported registry data. Indeed, insulintreated patients had substantially higher unadjusted mortality risk, which became weaker after multiple adjustments, indicating that a large part of the excess risk can be explained by comorbidities and complications. However, after extensive adjustments the excess risk still remained and was at about the same level as in the combination treatment group, as clearly shown in Fig. 1b. Further adjustments for creatinine clearance and microalbuminuria did not further attenuate the risk.

To date, no randomised controlled trial testing different glucose-lowering regimens in patients with CAD have convincingly demonstrated insulin-based therapy to be inferior to non-insulin-based therapy. The Bypass Angioplasty 
Revascularization Investigation 2 Diabetes trial randomised 2,368 patients with type 2 diabetes and CAD to either prompt revascularisation or intensive medical therapy alone; and to either insulin-provision or insulin-sensitisation therapy. Insulin provision and insulin sensitisation had similar 5 year mortality rates, $12.1 \%$ and $11.8 \%$, respectively ( $p=$ 0.89 ). Also, the rates of major cardiovascular events did not differ significantly among the groups [24]. However, these data are somewhat difficult to interpret with respect to the safety of insulin treatment, as almost $30 \%$ of the patients in the insulin-sensitisation arm were treated with insulin. A randomised trial of insulin treatment in patients with $C A D$ is needed to solve the safety aspects of insulin in these patients and the ongoing Outcome Reduction With Initial Glargine Intervention trial may provide further information on this topic [25].

Apart from the impact of glucose-lowering treatment, the present report confirms that previous well-known risk factors for mortality, such as smoking, myocardial damage, severity of coronary disease and renal complications, are strong risk factors in patients with diabetes. Clinical information on glucose-lowering therapy can now be added to this information - cardiologists and diabetologists should be aware that patients with CAD and type 2 diabetes treated with insulin are high-risk individuals, such that at only 4.1 years after an angiography, regardless of indication, $28 \%$ of individuals in our analysis had died.

\section{Limitations}

The present report is based on retrospective registry analysis and although substantial adjustments were made for potentially confounding variables, the effect of unidentified confounders cannot be excluded. However, adjustment was made for variables often related to insulin use, such as high $\mathrm{HbA}_{1 \mathrm{c}}$, long diabetes duration and retinopathy, as well as previous cardiovascular disease, such as heart failure. Some data from participating centres may vary slightly in accuracy, but variables on diabetes complications and angiography findings have been shown to be robust. Using an epidemiological definition of type 1 diabetes, we cannot exclude that some patients with type 1 diabetes were included in the cohort, particularly in the insulin-treated group. However, we consider that few patients with type 1 diabetes were included. About $5 \%$ of the CAD population are judged to have type 1 diabetes [26]. No information on treatment after angiography was available and the impact of changed diabetes treatment and other secondary preventive treatments after angiography could have influenced the results. On the other hand, it is less likely that patients receiving insulin treatment stopped this treatment and, regarding secondary prevention in CAD, a majority of patients with diabetes mellitus receive treatment according to guidelines in
Sweden [27]. No information on objective signs of heart failure (e.g. left ventricular ejection fraction) was available in the two registries. However, adjustments were made for hypertension, previous hospitalisation for heart failure and previous myocardial infarction, revascularisation and extent of $\mathrm{CAD}$, which are well-known risk factors for myocardial dysfunction.

In conclusion, patients with type 2 diabetes treated with insulin, either alone or in combination with oral glucoselowering drugs, are at increased risk for mortality after coronary angiography. This can, at least to a large extent, be explained by comorbidities and diabetes complications, but we cannot exclude the possibility that exogenous insulin might be unfavourable in these patients. As this is not a randomised trial, and despite our extensive efforts to adjust for important comorbidities, the results could still be explained by residual confounding. Further research on the effect of insulin treatment in patients with type 2 diabetes and CAD is needed to clarify this question.

Acknowledgements We would like to thank M. Miftaraj at the Centre of Registers in Gothenburg for statistical support.

Funding The Swedish Heart-Lung Foundation, the Swedish Federation of County Councils, the Swedish National Board of Health and Welfare and the Swedish Society of Cardiology and Diabetology supported this study and the registries.

Duality of interest The authors declare that there is no duality of interest associated with this manuscript. Several authors have received speaking honoraria from several pharmaceutical companies.

Contribution statement All the authors have made substantial contributions to this paper, having discussed and planned the aims and the methods and interpreted the results. PP and AN finalised the manuscript after adjustments from all the authors. BL performed the statistical analyses and AS merged the databases. All the authors have read and approved the final manuscript.

\section{References}

1. Yusuf S, Hawken S, Ounpuu S et al (2004) Effect of potentially modifiable risk factors associated with myocardial infarction in 52 countries (the INTERHEART study): case-control study. Lancet 364:937-952

2. Malmberg K, Yusuf S, Gerstein HC et al (2000) Impact of diabetes on long-term prognosis in patients with unstable angina and nonQ-wave myocardial infarction: results of the OASIS (Organization to Assess Strategies for Ischemic Syndromes) Registry. Circulation 102:1014-1019

3. Norhammar A, Lagerqvist B, Saleh N (2010) Long-term mortality after PCI in patients with diabetes mellitus: results from the Swedish Coronary Angiography and Angioplasty Registry. EuroIntervention 5:891-897 
4. Banning AP, Westaby S, Morice MC et al (2010) Diabetic and nondiabetic patients with left main and/or 3-vessel coronary artery disease: comparison of outcomes with cardiac surgery and paclitaxel-eluting stents. J Am Coll Cardiol 55:1067-1075

5. Holman RR, Paul SK, Bethel MA, Matthews DR, Neil HA (2008) 10-year follow-up of intensive glucose control in type 2 diabetes. N Engl J Med 359:1577-1589

6. Patel A, MacMahon S, Chalmers J et al (2008) Intensive blood glucose control and vascular outcomes in patients with type 2 diabetes. N Engl J Med 358:2560-2572

7. Gerstein HC, Miller ME, Byington RP et al (2008) Effects of intensive glucose lowering in type 2 diabetes. N Engl J Med 358:2545-2559

8. Duckworth W, Abraira C, Moritz T et al (2009) Glucose control and vascular complications in veterans with type 2 diabetes. $\mathrm{N}$ Engl J Med 360:129-139

9. Ray KK, Seshasai SR, Wijesuriya S et al (2009) Effect of intensive control of glucose on cardiovascular outcomes and death in patients with diabetes mellitus: a meta-analysis of randomised controlled trials. Lancet 373:1765-1772

10. Mellbin LG, Malmberg K, Norhammar A, Wedel H, Ryden L (2008) The impact of glucose lowering treatment on long-term prognosis in patients with type 2 diabetes and myocardial infarction: a report from the DIGAMI 2 trial. Eur Heart J 29:166-176

11. Gudbjornsdottir S, Cederholm J, Nilsson PM, Eliasson B (2003) The National Diabetes Register in Sweden: an implementation of the St. Vincent Declaration for Quality Improvement in Diabetes Care. Diabetes Care 26:1270-1276

12. Hoelzel W, Weykamp C, Jeppsson JO et al (2004) IFCC reference system for measurement of hemoglobin A1c in human blood and the national standardization schemes in the United States, Japan, and Sweden: a method-comparison study. Clin Chem 50:166-174

13. Anselmino M, Ohrvik J, Malmberg K, Standl E, Ryden L (2008) Glucose lowering treatment in patients with coronary artery disease is prognostically important not only in established but also in newly detected diabetes mellitus: a report from the Euro Heart Survey on Diabetes and the Heart. Eur Heart J 29:177-184

14. McGuire DK, Newby LK, Bhapkar MV et al (2004) Association of diabetes mellitus and glycemic control strategies with clinical outcomes after acute coronary syndromes. Am Heart J 147:246252

15. Malmberg K, Ryden L, Wedel H et al (2005) Intense metabolic control by means of insulin in patients with diabetes mellitus and acute myocardial infarction (DIGAMI 2): effects on mortality and morbidity. Eur Heart J 26:650-661

16. Kosiborod M, Inzucchi SE, Krumholz HM et al (2008) Glucometrics in patients hospitalized with acute myocardial infarction: defining the optimal outcomes-based measure of risk. Circulation 117:1018-1027

17. Pinto DS, Kirtane AJ, Pride YB et al (2008) Association of blood glucose with angiographic and clinical outcomes among patients with ST-segment elevation myocardial infarction (from the CLARITY-TIMI-28 study). Am J Cardiol 101:303-307

18. Pinto DS, Skolnick AH, Kirtane AJ et al (2005) U-shaped relationship of blood glucose with adverse outcomes among patients with ST-segment elevation myocardial infarction. J Am Coll Cardiol 46:178-180

19. Svensson AM, McGuire DK, Abrahamsson P, Dellborg M (2005) Association between hyper- and hypoglycaemia and 2 year allcause mortality risk in diabetic patients with acute coronary events. Eur Heart J 26:1255-1261

20. Kosiborod M, Inzucchi SE, Goyal A et al (2009) Relationship between spontaneous and iatrogenic hypoglycemia and mortality in patients hospitalized with acute myocardial infarction. JAMA 301:1556-1564

21. Mellbin LG, Malmberg K, Waldenstrom A, Wedel H, Ryden L (2009) Prognostic implications of hypoglycaemic episodes during hospitalisation for myocardial infarction in patients with type 2 diabetes: a report from the DIGAMI 2 trial. Heart 95:721-727

22. Arcaro G, Cretti A, Balzano S et al (2002) Insulin causes endothelial dysfunction in humans: sites and mechanisms. Circulation 105:576-582

23. Angiolillo DJ, Bernardo E, Ramirez C et al (2006) Insulin therapy is associated with platelet dysfunction in patients with type 2 diabetes mellitus on dual oral antiplatelet treatment. J Am Coll Cardiol 48:298-304

24. Frye RL, August $\mathrm{P}$, Brooks MM et al (2009) A randomized trial of therapies for type 2 diabetes and coronary artery disease. N Engl J Med 360:2503-2515

25. Gerstein H, Yusuf S, Riddle MC, Ryden L, Bosch J (2008) Rationale, design, and baseline characteristics for a large international trial of cardiovascular disease prevention in people with dysglycemia: the ORIGIN Trial (Outcome Reduction with an Initial Glargine Intervention). Am Heart J 155:26-32, 32 e21-26

26. James S, Angiolillo DJ, Cornel JH et al (2010) Ticagrelor vs. clopidogrel in patients with acute coronary syndromes and diabetes: a substudy from the PLATelet inhibition and patient Outcomes (PLATO) trial. Eur Heart J 31:3006-3016

27. Norhammar A, Lindback J, Ryden L, Wallentin L, Stenestrand U (2007) Improved but still high short- and long-term mortality rates after myocardial infarction in patients with diabetes mellitus: a time-trend report from the Swedish Register of Information and Knowledge about Swedish Heart Intensive Care Admission. Heart 93:1577-1583 